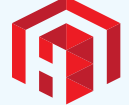 Heighten Science \\ P U B L I C I T I O N S Corporation \\ ISSN \\ 2575-0143}

*Address for Correspondence: $\mathrm{CE}$

Papadopoulos, Grigoriou E 11, 55236,

Thessaloniki, Greece, Tel/fax: 00302310

223330; Email: chpapado@auth.gr

Submitted: 17 October 2016

Approved: 05 December 2016

Published: 07 December 2016

Copyright: @2016 Papadopoulos et al. This is an open access article distributed under the Creative Commons Attribution License, which permits unrestricted use, distribution, and reproduction in any medium, provided the original work is properly cited.

Keywords: Left atrium; Remodeling; Myocardial infarction; Diastolic function; BNP

\title{
Left Atrial Remodeling is Associated with Left Ventricular Remodeling in Patients with Reperfused Acute Myocardial Infarction
}

\author{
Christodoulos E. Papadopoulos ${ }^{1 *}$, Dimitrios G. Zioutas ${ }^{1}$, \\ Panagiotis Charalambidis ${ }^{1}$, Aristi Boulbou ${ }^{1}$, Konstantinos \\ Triantafyllou ${ }^{1}$, Konstantinos Baltoumas ${ }^{1}$, Haralambos I. \\ Karvounis ${ }^{2}$ and Vassilios Vassilikos ${ }^{1}$ \\ ${ }^{1} 3^{\text {rd }}$ Department of Cardiology, Hippokrateio University Hospital, Aristotle University of \\ Thessaloniki, Greece \\ ${ }^{2} 1^{\text {st }}$ Department of Cardiology, AHEPA University Hospital, Aristotle University of Thessaloniki, \\ Greece
}

\section{ABSTRACT}

Background: Left atrial volume (LAV) has been established as a sensitive marker of left ventricular (LV) diastolic function and as an independent predictor of mortality in patients with acute myocardial infarction (AMI). LA remodeling and its determinants in the setting of AMI have not been much studied.

Methods: We studied 53 patients with anterior AMI and a relatively preserved LV systolic function, who underwent complete reperfusion and received guidelines guided antiremodeling drug management. LA and LV remodeling were assessed using $2 \mathrm{D}$ echocardiography at baseline and 6 months. LAV indexed for BSA (LAVi) was used as the index of LA size and further LA remodeling.

Results: LAVi increased significantly at 6 months compared to baseline [28.1 (23.0-34.5) vs 24.4 (19.5$31.6) \mathrm{ml} / \mathrm{m} 2, \mathrm{p}=0.002$ ] following LV end diastolic-volume index change [56.8 (47.6-63.9) vs $49.5(42.0-58.4) \mathrm{ml} /$ $\mathrm{m} 2, \mathrm{p}=0.0003$ ]. Other standard LV diastolic function indices did not show any significant change. Univariate analysis showed a strong positive correlation of LAVi change with BNP levels at discharge, LV mass index and LV volumes indices change, throughout the follow up period. Multivariate regression analysis revealed that BNP plasma levels was the most important independent predictor of $L A$ remodeling ( $b-c o e f .=0.630, p=0.001$ ).

Conclusions: Despite current antiremodeling strategies in patients with AMI, LA remodeling is frequently asssociated with LV remodeling. Additionally LAVi change in the mid-term reflects better than standard echocardiographic indices LV diastolic filling impairment.

\section{INTRODUCTION}

There is increasing evidence that left atrial volume (LAV) is a more robust marker of cardiovascular events than LA area or diameter in subjects with sinus rhythm [1]. In more detail LAV has been strongly associated with mortality in high risk populations, such as patients with acute myocardial infarction [2-4], dilated cardiomyopathy [5], atrial arrhythmias [6] and in patients undergoing mitral valve surgery [7]. Furthermore LAV has been associated with the development of atrial fibrillation, ischemic stroke and heart failure $[1,8,9]$ and is considered a stable indicator of $\mathrm{LV}$ diastolic dysfunction reflecting better its severity and chronicity [10]. 
Although LV remodeling has been well described as a potential complication of myocardial infarction (MI), LA remodeling has not been studied in detail [11]. The aim of the present study was to evaluate the prevalence of LA remodeling in patients with acute MI undergoing effective reperfusion and receiving up to date antiremodeling drug management.

\section{METHODS}

The study was conducted at AHEPA University Hospital Cardiology Department and its protocol was approved by the Institutional Committee on human research. The study complied with the Declaration of Helsinki and all patients gave written inform consent before enrollment.

Sixty two consecutive patients were enrolled in the study. Patients were considered eligible if they presented with an acute anterior wall ST elevation MI, with a angiographically documented left anterior descending coronary artery stenosis (culprit lesion) and no history of cardiovascular disease. Acute MI was defined according to published guidelines [12]. All patients were successfully reperfused with either thrombolysis, primary percutaneous coronary intervention (PCI), thrombolysis followed by PCI within 24 hours of admission depending on the catheterization lab availability or coronary artery bypass grafting (CABG). Patients had to have TIMI flow grade III in LAD, following successful lysis or following PCI with stent deployment. Failure to achieve a TIMI flow grade III consisted an exclusion criterion. Additionally, patients that had significant coronary artery stenoses (lesion $>70 \%$ ) involving different myocardial wall territories and were left untreated, were excluded from the study population. Furthermore patients were excluded from the study if they had a poor echocardiographic image, any history of coronary heart disease, any history of terminal or systematic disease, any severe valve disease or cardiomyopathy or absence of sinus rhythm. During enrollment 16 patients with reperfused anterior wall STEMI were excluded from the study according to relevant inclusion and exclusion criteria. Ten patients had significant coronary artery stenoses that were left untreated by the interventionalist, 2 developed atrial fibrillation and 4 had inadequate echocardiographic images.

All patients received a complete antiremodeling and secondary prevention drug management including dual antiplatelet therapy, ACE inhibitors, b-blockers and statins where no contraindicated. Every effort was made in order to improve patients adherence to the recommended treatment.

Blood samples for measuring CPK MB levels, were taken on admission, then every 8 hours for the first day and subsequently every morning till discharge and peak value was reported. In addition B-type Natriuretic Peptide (BNP) levels at discharge were assessed using a commercially available immunofluoresence assay (BNP Triage, Biosite, San Diego).

All patients underwent a comprehensive transthoracic echocardiographic study using a GE Vingmed Vivid 7 system (GE Vingmed Ultrasound AS, Horten, Norway), at the day of discharge.

LV (end-systolic and end-diastolic) volumes and ejection fraction were estimated using Simpson's modified biplane method and were further corrected for body surface area (end-systolic and end-diastolic volume index) [13]. The wall motion score index (WMSi) was obtained semiquantitatively using a 16-segment division of the LV [13]. LA volume was estimated using the biplane Simpson's method from the apical 4 and 2 chamber views and was further corrected for body surface area to derive the LAV index (LAVi) [14]. To address the presence of LV hypertrophy LV mass index was assessed as previously described [15]. 
Pulsed Doppler echocardiography in order to assess standard LV diastolic filling velocities was performed using the apical 4-chamber view. Peak early filling velocity (E wave) and E wave deceleration time (DT) were recorded [16,17]. Color TDI was used to assess mitral annulus velocities. A 5-mm sample volume was placed at the apical 4-chamber view on the septal and lateral corner of the mitral annulus and the early diastolic velocity (e') was recorded. The velocities obtained by the two different points of the mitral annulus were averaged. Furthermore the ratio of early transmitral filling velocity (E wave) to early diastolic mitral annulus velocity (LV E/e' index) was calculated [18]. Finally propagation velocity was obtained and the ratio of $\mathrm{E}$ wave to propagation velocity was calculated (E/Vp) [19].

All echocardiographic measurements were obtained by a single experienced operator (C.E.P.) from three different cardiac cycles and were further averaged. All measurements were processed offline. The follow-up was undertaken at 6 months when a complete echocardiographic study was repeated.

Statistical analysis was performed with SPSS version 15.0 (SPSS Inc). A p-value less than 0.05 was considered statistically significant. Descriptive statistics for continuous

Table 1: Study cohort characteristics $(n=53)$.

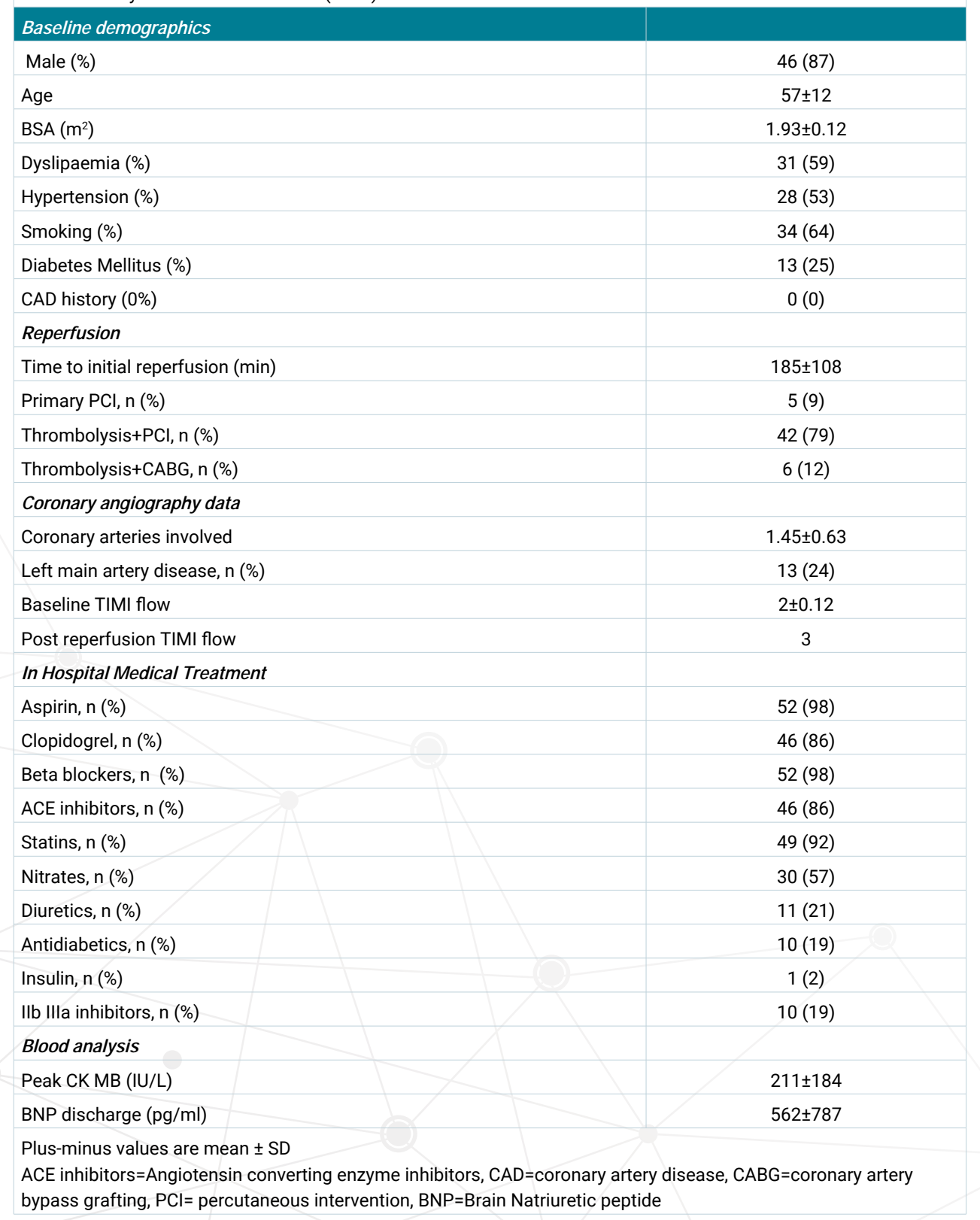


variables are presented as median \pm interquartile range and for nominal or ordinal variables as absolute frequencies and percentages. Descriptive statistics for the changes in LAVI and EDVI are also presented as mean $\pm 95 \%$ confidence interval, since they followed the normal distribution. Comparison of variables was done with the use the Wilcoxon signed ranks test (for continuous variables) and the McNemar test (for nominal or ordinal dichotomous variables), whichever was appropriate. Independent correlates of the LA volume index change throughout the follow-up were identified by their respective correlation coefficient. Variables significant at a $p$ value of less than 0.01 were entered in the multivariate linear regression model and selection was based on the strength of correlation and its significance level.

\section{RESULTS}

From 62 patients initially enrolled in the study 53 consisted the study population. Eight were excluded due to poor echocardiographic images at follow up and one patient was lost to follow up. The baseline characteristics of the cohort are presented in Table 1. The population consisted mainly of middle aged males. Of note, there was a high prevalence of the five major cardiovascular risk factors. Nineteen patients had multivessel disease. Thrombolysis followed by PCI was the most commonly employed reperfusion therapy (79\% of all patients) and the median time to reperfusion was 2.5 hours. TIMI III flow was evidenced in all patients except the ones treated with CABG where post surgery angiography was not performed due to ethical reasons.

Most patients received a combination therapy of the drugs used for secondary prevention showing significant adherence through follow-up period. Overall, 36 patients $(78 \%)$ at baseline and 38 patients $(83 \%)$ at follow-up were receiving an antiplatelet, a $\beta$-blocker, an ACE inhibitor, and a statin.

The echocardiographic variables at baseline and follow-up are presented in Table 2. All volumes assessed (LAVi, LVEDVi, LVESVi) increased significantly throughout the follow-up period. Indeed, LAVi showed a mean increase of 16.78\% (95\% CI for mean 7.95\%-25.61\%) and similarly LV EDVi a mean increase of 15.69\% (95\% CI for mean 5.63\%-24.61\%). Previous studies have used an increase in baseline LV end-diastolic volume $>20 \%$ at follow-up as a cutoff value of major LV remodeling [16,20]. By using the same cutoff value for both LA ( $>20 \%$ increase in baseline LA volume) and LV ( $>20 \%$ increase in baseline LV end-diastolic volume) remodeling, we evidenced a significant prevalence of these geometric alterations, of 35\% and $43 \%$ respectively. Interestingly patients who developed LA remodeling ( $>20 \%$ increase in baseline LA volume) had higher plasma BNP levels at baseline compared to patients without evidence of LA remodeling at follow-up (716 \pm 926 vs $312 \pm 404 \mathrm{pg} / \mathrm{ml}, \mathrm{p}=0.005)$. Additionally we found that LA volume index at baseline was relatively high compared to the previously reported normal value for the general population $\left(22 \pm 6 \mathrm{ml} / \mathrm{m}^{2}\right)$ [21]. The proportion of patients with a LAVi larger than the $32 \mathrm{ml} / \mathrm{m}^{2}$ was $22 \%$ at baseline and $33 \%$ at follow-up. In terms of diastolic function assessment except LAVi, there was no change in the specific diastolic function indices that were assessed between baseline and follow-up (Table 2).

Univariate linear regression analysis was undertaken in order to highlight the variables that were associated with LA remodeling (any change in LAVi). As shown in Table 3, LV volumes, their net change throughout the follow-up period and LV hypertrophy (LV mass index) were positively correlated with LA remodeling, while baseline LV EF was negatively correlated. There was no association with any of the other LV diastolic indices tested. Finally there was a significant positive correlation with plasma BNP levels. Multivariate regression analysis revealed that BNP levels are the most significant predictor of LA remodeling in this specific cohort (Table 3). 
Table 2: Echocardiographic variables at baseline and at follow-up.

\begin{tabular}{|c|c|c|c|}
\hline Variable & Baseline & 6 month follow-up & p-value \\
\hline \multicolumn{4}{|l|}{ Remodeling } \\
\hline $\begin{array}{l}\text { LV remodeling } \\
(>20 \% \text { increase in EDVi) }\end{array}$ & & $23(43)$ & \\
\hline $\begin{array}{l}\text { LA remodeling } \\
(>20 \% \text { increase in LAVi) }\end{array}$ & & $19(35)$ & \\
\hline LAVI $\left(\mathrm{ml} / \mathrm{m}^{2}\right)$ & $24.4(19.5-31.6)$ & $28.1(23.0-34.5)$ & 0.002 \\
\hline LVESVi $\left(\mathrm{ml} / \mathrm{m}^{2}\right)$ & $24.7(20.6-31.3)$ & $26.5(19.9-37.4)$ & 0.017 \\
\hline LVEDVi $\left(\mathrm{ml} / \mathrm{m}^{2}\right)$ & $49.5(42.0-58.4)$ & $56.8(47.6-63.9)$ & 0.0003 \\
\hline \multicolumn{4}{|l|}{ LV Systolic function } \\
\hline LVEF (\%) & $47.3(39.2-57.0)$ & $50.3(39.2-56.7)$ & ns \\
\hline WMSi & $1.68(1.42-1.93)$ & $1.43(1.25-1.89)$ & 0.006 \\
\hline \multicolumn{4}{|l|}{ LV Diastolic function } \\
\hline E wave $(\mathrm{cm} / \mathrm{sec})$ & $0.76(0.68-0.95)$ & $0.80(0.65-0.98)$ & ns \\
\hline$E / A$ & $1.18(0.83-1.38)$ & $1.13(0.73-1.35)$ & ns \\
\hline DT (msec) & $236.5(170.3258 .5)$ & $237.5(188.5-263.5)$ & ns \\
\hline $\mathrm{E} / \mathrm{Em}$ & $17.4(13.6-21.1)$ & $16.2(12.4-18.9)$ & ns \\
\hline$E / V p$ & $1.48(1.17-2.24)$ & $1.46(1.04-2.12)$ & ns \\
\hline $\mathrm{Vp}(\mathrm{cm} / \mathrm{s})$ & $50.0(38.5-66.8)$ & $51.0(40.0-67.0)$ & ns \\
\hline
\end{tabular}

Values are median (interquartile range) or $\mathrm{n}(\%)$

$D T=E$ Wave Deceleration Time, $E=E$ wave velocity, $E / A=E$ to $A$ wave velocity, $E / E m=E$ wave velocity to mitral annulus early diastolic velocity ratio, $E / \mathrm{Vp}=\mathrm{E}$ wave velocity to propagation velocity, $\mathrm{LAVi}=$ left atrial volume index, $L V E D V i=l$ feft ventricular end-diastolic volume index, LVESVi=left ventricular end-systolic volume index, LVEF=left Ventricular Ejection Fraction, $\mathrm{Vp}=$ Propagation velocity, WMSi=Left Ventricular Wall Motion Score index

Table 3: Univariate and multivariate linear regression analysis for left atrial remodeling $\left(\mathrm{R}^{2}\right.$ for the multivariate model was 0.727$)$.

\begin{tabular}{|c|c|c|c|c|}
\hline Variable (units) & $\begin{array}{c}\text { Univariate correlation } \\
\text { coefficient }\end{array}$ & p-value & $\begin{array}{c}\text { Multivariate beta } \\
\text { coefficient }\end{array}$ & p-value \\
\hline$\Delta$ LV EDVi $\left(\mathrm{ml} / \mathrm{m}^{2}\right)$ & 0.357 & 0.016 & 0.275 & $\mathrm{~ns}$ \\
\hline$\Delta$ LV ESVi $\left(\mathrm{ml} / \mathrm{m}^{2}\right)$ & 0.407 & 0.006 & 0.014 & $\mathrm{~ns}$ \\
\hline LV EF $(\%)$ & -0.386 & 0.009 & -0.339 & $\mathrm{~ns}$ \\
\hline Mass index $\left(\mathrm{gr} / \mathrm{m}^{2}\right)$ & 0.322 & 0.03 & & \\
\hline DT $(\mathrm{msec})$ & 0.189 & 0.220 & & \\
\hline Em $(\mathrm{cm} / \mathrm{sec})$ & -0.281 & 0.061 & & 0.001 \\
\hline BNP $(\mathrm{pg} / \mathrm{ml})$ & 0.503 & 0.003 & 0.630 & 0 \\
\hline
\end{tabular}

$\mathrm{BNP}=$ Brain Natriuretic Peptide, DT=E Wave Deceleration Time, Em=mitral annulus early diastolic velocity, LVEDVi=left ventricular end-diastolic volume index, LVESVi=left ventricular end-systolic volume index, LVEF=left Ventricular Ejection Fraction, Mass index=left ventricular mass index, WMSi=Left Ventricular Wall Motion Score index, $\Delta=$ net change throughout follow-up

\section{DISCUSSION}

The present study shows that despite the widespread use of reperfusion and antiremodeling drug strategy in patients with acute MI, LA remodeling following LV remodeling in patients with a relatively preserved LV systolic function, is a common sequale post MI. Furthermore we evidenced that in this specific population, LA volume was the only LV diastolic function index that was significantly impaired in the midterm and this LA remodeling process could be best predicted by BNP plasma levels at discharge.

Although the role of LA in the setting of acute MI has been well recognized from a long time ago [22], only the last decade LA volume has emerged as an independent predictor of mortality in this setting [2,3]. Additionally, LA enlargement has been proposed as a barometer of diastolic function and a predictor of common cardiovascular outcomes 
such as atrial fibrillation, stroke, congestive heart failure, and cardiovascular death in various clinic entities [2-9]. Therefore, enlargement of the LA reflects remodeling associated with serious pathophysiologic processes.

Doppler indices have been well studied and validated in AMI populations $[16,18,19]$ and reflect filling pressures at the time of measurement. LA volume on the other hand represents the cumulative effects of filling pressures over time. Therefore, LA volume is regarded as a more stable parameter representing chronic LV diastolic function. Our study showed that in patients with a relatively preserved $\mathrm{LV}$ ejection fraction post AMI, between all standard LV diastolic function indices, LA volume was the only one that showed significant change in the midterm, supporting the concept that this index is a more real-life diastolic function index. In our study population LA volume index at baseline was relatively high compared to the previously reported normal value for the general population $\left(22 \pm 6 \mathrm{ml} / \mathrm{m}^{2}\right)$ [21]. This finding was probably the result of the relative high prevalence of arterial hypertension (53\%) of the cohort, the population mean age $(57 \pm 12)$ and LV hypertrophy.

Previous studies have evidenced the occurrence of LA remodeling and established the close relation between LA and LV remodeling following acute MI [11]. In the present study we documented a quite similar proportion of \% change of LAVi and LVEDVi to follow-up, reflecting the similar natural history of these two different left chamber geometric alterarions. By using the cutoff value of $20 \%$ as described in the results section, we found a higher proportion of patients developing LV remodeling than LA remodeling ( 43 vs $35 \%$ ). The main reason for that could be that despite the fact that increased LV filling pressures are the main mechanical driving forces for LA and $\mathrm{LV}$ remodeling, $\mathrm{LV}$ is additionally affected by direct myocardial injury and further ischemia. Furthermore LA usually needs more time to adapt to high LV filling pressures than $\mathrm{LV}$, as evidenced in patients with mitral valve regurgitation. In contrast to our findings there are some reports, where no association between LA volume and further $\mathrm{LV}$ remodeling was found post $\mathrm{MI}$ and that might probably be due to the relative short follow-up period [23].

In the present study we evidenced that BNP plasma levels at baseline are the most significant independent predictor of LA remodeling in the midterm. BNP has been well recognized as an independent predictor of LV remodeling and other adverse cardiovascular events following acute MI [24-27]. Additionally this marker has been associated with various diastolic function indices reflecting LV filling pressure and has been shown to give incremental prognostic information in the same setting [28]. Although theoretically BNP levels would normally have some association with LA volume, as both indices are considered surrogates of LV diastolic filling properties, convincing data in the literature are missing. In contrast there are several reports where BNP levels are correlated with LA volume in various other clinical entities and especially patients with heart failure [29-31]. We have evidenced a strong positive association between LA remodeling and LV hypertrophy as expressed by LV mass index. It has been shown and we have published in the past similar results in such cohorts, documenting this strong association between LV hypertrophy and LV remodeling following an acute MI [26]. The present study establishes the correlation of LA remodeling with LV mass, reinforcing our concept that LA remodels together with $\mathrm{LV}$ and that the driving forces are quite the same.

The main limitation of the study was the small population, and our findings need to be replicated in larger cohorts. Another issue concerns the homogeneity of the cohort, as reperfusion treatment included lysis, PCI or CABG. It might be more appropriate to exclude CABG patients due to the fact that we have not angiographically documented graft patency post operation. In any case all patients had an anterior wall AMI with a relatively preserved LV systolic performance and received reperfusion with no 
evidence of any major adverse cardiovascular events through the follow-up time. This fact indirectly shows that probably there was no residual ischemia in the study population. Finally there is concern about the time (chronicity) of follow-up that might influence our results. There is no consensus about the exact period that LA needs to remodel following an acute MI, although many of the studies in the literature evaluating mainly LV remodeling have used the same 6 months period.

The clinical implications of our findings could be the increased awareness that physicians should show in patients with evidence of evolving LA remodeling on top of LV remodeling following an AMI. In this specific population up regulation of antiremodeling medication, increased adherence and close follow up with possible BNP plasma evaluation, might change the course of the disease. A prognostic assessment in this specific cohort, evaluating the impact of LA volume change on clinical endpoints, like major cardiovascular events or rehospitalizations would be interesting, although it is of no doubt that this study is underpowered to show any such association.

In conclusion, we have documented evidence that remodeling involves LA in addition to the LV chamber in a significant proportion of patients following an acute MI. Between other standard LV diastolic function indices, LA volume change stands as the only index that consistently deteriorates in the mid-term following an AMI and is strongly associated with BNP levels at discharge.

\section{REFERENCES}

1. Tsang TS, Abhayaratna WP, Barnes ME, Miyasaka Y, Gersh BJ, et al. Prediction of cardiovascular outcomes with left atrial size. Is volume superior to area or diameter? J Am Coll Cardiol. 2006; 47: 10181023. Ref.: https://goo.gl/yCLjTS

2. Moller JE, Hillis GS, Oh JK, Seward JB, Reeder GS, et al. Left atrial volume: A powerful predictor of survival after acute myocardial infarction. Circulation. 2003; 107: 2207-2212. Ref.: https://goo.gl/CmD3VU

3. Beinart R, Boyko V, Schwammenthal E, Kuperstein R, Sagie A, et al. Long-term prognostic significance of left atrial volume in acute myocardial infarction. J Am Coll Cardiol. 2004; 44: 327-334. Ref.: https://goo.gl/eTmeb6

4. Meris A, Amigoni M, Uno $\mathrm{H}$, Thune $\mathrm{JJ}$, Verma A, et al. Left atrial remodeling in patients with myocardial infarction complicated by heart failure, left ventricular dysfunction, or both: the VALIANT Echo Substudy. Eur Heart J. 2009; 30: 56-65. Ref.: https://goo.gl/qJ OsLt

5. Rossi A, Cicoira M, Zanolla L, Sandrini R, Golia G, et al. Determinants and prognostic value of left atrial volume in patients with dilated cardiomyopathy. J Am Coll Cardiol. 2002; 40: 1425-1430. Ref.: https://goo.gl/ITIICZ

6. Cabin HS, Clubb KS, Hall C, Perlmutter RA, Feinstein AR. Risk for systemic embolization of atrial fibrillation without mitral stenosis. Am J Cardiol. 1990; 65: 1112-1116. Ref.: https://goo.gl/mn9lcr

7. Reed $\mathrm{D}$, Abbott RD, Smucker ML, Kaul S. Prediction of outcome after mitral valve replacement in patients with symptomatic chronic mitral regurgitation. The importance of left atrial size. Circulation. 1991; 84: 23-34. Ref.: https://goo.gl/SPHSnd

8. Barnes ME, Miyasaka Y, Seward JB, Gersh BJ, Rosales AG, et al. Left atrial volume in the prediction of first ischemic stroke in an elderly cohort without atrial fibrillation. Mayo Clin Proc. 2004; 79: 1008-1014. Ref.: https://goo.gl/p4J RTV

9. Gottdiener JS, Kitzman DW, Aurigemma GP, Arnold AM, Manolio TA. Left atrial volume, geometry, and function in systolic and diastolic heart failure of persons $>65$ years of age (the Cardiovascular Health Study). Am J Cardiol. 2006; 97: 83-89. Ref.: https://goo.gl/dUZfOT

10. Nagueh SF, Appleton CP, Gillebert TC, Marino PN, Oh JK, et al. Recommendations for the evaluation of left ventricular diastolic function by echocardiography. J Am Soc Echocardiogr. 2009; 22: 107-133. Ref.: https://goo.gl/wMiutw

11. Popescu BA, Macor F, Antonini-Canterin F, Giannuzzi P, Temporelli PL, et al. Left atrial remodelling after acute myocardial infarction (results of the GISSI-3 Echo substudy). Am J Cardiol. 2004; 93: 1156-1159. Ref.: https://goo.gl/vNcq9r

12. Kushner FG, Hand M, Smith SC Jr, King SB III, Anderson JL, et al. 2009 focused update of the ACC/AHA guidelines for the management of patients with ST-Elevation myocardial infarction (updating the 2004 guideline and 2007 focused update) and the ACC/AHA/SCAI guidelines on percutaneous coronary intervention (updating the 2005 guideline and 2007 focused update): a report of the American College of Cardiology Foundation/American Heart Association Task Force on Practice Guidelines. J Am Coll Cardiol. 2009; 54 2205-2241. Ref.: https://goo.gl/gDx47k 
13. Schiller NB, Shah PM, Crawford M, De Maria A, Devereux R, et al. American Society of Echocardiography Committee on Standards on Quantitation of Two-Dimensional Echocardiograms: recommendations for quantitation of the left ventricle by two-dimensional echocardiography. J Am Soc Echocardiogr. 1989; 2: 358-367. Ref.: https://goo.gl/tio1Hp

14. Lang RM, Bierig M, Devereux RB, Flachskampf FA, Foster E, et al. American Society of Echocardiography's Nomenclature and Standards Committee; Task Force on Chamber Quantification; American College of Cardiology Echocardiography Committee; American Heart Association; European Association of Echocardiography, European Society of Cardiology recommendations for chamber quantification. Eur J Echocardiogr. 2006; 7: 79-108. Ref.: https://goo.gl/WajRIK

15. Jilaihawi H, Greaves S, Rouleau JL, Pfeffer MA, Solomon SD. for the Healing and Early Afterload 47. Reducing Therapy (HEART) Trial Investigators. Am J Cardiol. 2003; 91: 723-726. Ref.: https://goo.gl/9UYX0m

16. Temporelli PL, Giannuzzi P, Nicolosi GL, Latini R, Franzosi MG, et al. Doppler-derived mitral deceleration time as a strong prognostic marker of left ventricular remodeling and survival after acute myocardial infarction. Results of the GISSI-3 echo substudy. J Am Coll Cardiol. 2004; 43: 1646-1653. Ref.: https://goo.gl/tPVsKn

17. Appleton CP, Jensen JL, Hatle LK, Oh JK. Doppler evaluation of left and right ventricular diastolic function: a technical guide for obtaining optimal flow velocity recordings. J Am Soc Echocardiogr. 1997; 10: 271-292. Ref.: https://goo.gl/KApYZI

18. Moller JE, Pellicka PA, Hillis GS, Oh JK. Prognostic importance of diastolic function and filling pressure in patients with acute myocardial infarction. Circulation. 2006; 114: 438-444. Ref.: https://goo.gl/ylpQqc

19. Moller JE, Sondergaard E, Seward JB, Appleton CP, Egstrup K. Ratio of left ventricular peak E-wave velocity to flow propagation velocity assessed by color M-mode Doppler echocardiography in first myocardial infarction. Prognostic and clinical implications. J Am Coll Cardiol. 2000; 35: 363-370. Ref.: https://goo.gl/Dns2tx

20. Savoye C, Equine O, Tricot O, Nugue O, Segrestin B, et al. Myocardial infarction in modern clinical practice (from the REmodelage Ventriculaire [REVE] Study Group). Am J Cardiol. 2006; 98: 1144-1149. Ref.: https://goo.gl/svzlxo

21. Abhayaratna WP, Seward JB, Appleton CP, Douglas PS, Oh JK, et al. Left atrial size. Physiologic determinants and clinical applications. J Am Coll Cardiol. 2006; 47: 2357-2363. Ref.: https://goo.gl/bHbGdl

22. Matsuda $\mathrm{Y}$, Toma $\mathrm{Y}$, Ogawa $\mathrm{H}$, Matsuzakl M, Katayama $\mathrm{K}$, et al. Importance of left atrial function in patients with myocardial infarction. Circulation. 1983; 67: 566-571. Ref.: https://goo.gl/EFOuiF

23. Hillis GS, Ujino K, Mulvagh S, Hagen M, Oh K. Echocardiographic indices of increased left ventricular filling pressure and dilation after acute myocardial infarction. J Am Soc Echocardiogr. 2006; 19: 450-456. Ref.: https://goo.gl/MWzYOO

24. Grilley JG, Farrer M. Left ventricular remodeling and brain natriuretic peptide after first myocardial infarction Heart. 2001; 86: 638-642. Ref.: https://goo.gl/G9ecjR

25. Nilsson JC, Groenning BA, Nielsen G, Fritz-Hansen T, Trawinski J, et al. Left ventricular remodeling in the first yea after acute myocardial infarction and the predictive value of $\mathrm{N}$-terminal pro brain natriuretic peptide. Am Heart $\mathrm{J}$. 2002; 143: 696-702. Ref.: https://goo.gl/6MJ 47d

26. Papadopoulos CE, Karvounis HI, Giannakoulas G, Karamitsos TD, Efthimiadis GK, et al. Predictors of left ventricular remodeling after reperfused acute myocardial infarction. Am J Cardiol. 2007; 99: 1024-1025. Ref.: https://goo.gl/0cwklK

27. De Lemos JA, Morrow DA, Bentley JH, Omland T, Sabatine MS, et al. The prognostic value of B-type natriuretic peptide in patients with acute coronary syndromes. N Engl J Med. 2001; 345: 1014-1021. Ref. https://goo.gl/psuw0]

28. Kruszewski K, Scott AE, Barclay JL, Small GR, Croal BL, et al. Noninvasive assessment of left ventricular filling pressure after acute myocardial infarction: A prospective study of the relative prognostic utility of clinical assessment, echocardiography, and B-type natriuretic peptide. Am Heart J. 2010; 159: 47-54. Ref.: https://goo.gl/0EQVpK

29. Tripepi G, Mattace-Raso, Mallamaci F, Benedetto FA, Witteman J, et al. Biomarkers of left atria volume. A longitudinal study in patients with end stage renal disease. Hypertension. $2009 ; 54: 818$ 824. Ref.: https://goo.gl/HDvfCh

30. Kim H, Jun DW, Cho YK, Nam CW, Han SW, et al. The correlation of left atrial volume index to the level of $\mathrm{N}$-terminal pro-BNP in heart failure with a preserved ejection fraction. Echocardiography. 2008; 5: 961-967. Ref.: https://goo.gl/tNPccc

31. Barclay JL, Kruszewski K, Croal BL, Cuthbertson BH, Oh JK, et al. Relation of left atrial volume to B-type natriuretic peptide levels in patients with stable chronic heart failure. Am J Cardiol. 2006; 98: 98-101. Ref. https://goo.gl/ROfLZn 